\title{
Analysis of Electrical and Thermal Models for Pulsed IMPATT Diode Simulation
}

\author{
ALEXANDER ZEMLIAK $^{1,2}$, EUGENE MACHUSKY ${ }^{2}$ \\ ${ }^{1}$ Department of Physics and Mathematics \\ Autonomous University of Puebla \\ Av. San Claudio y 18 Sur, Puebla, 72570 \\ MEXICO \\ ${ }^{2}$ Institute of Technical Physics \\ National Technical University of Ukraine \\ UKRAINE
}

\begin{abstract}
Some nonlinear models are presented for modeling and analyzing IMPATT high-power pulse diodes. These models are suitable for analyzing different operating modes of the oscillator. The first model is a precise one, which describes all important electrical phenomena on the basis of the continuity equations and Poisson's equation, and it is correct until $300 \mathrm{GHz}$. The second approximate mathematical model suitable for the analysis of IMPATT diode stationary operation oscillator and for optimization of internal structure of the diode. The temperature distribution in the semiconductor structure is obtained using the special thermal model of the IMPATT diode, which is based on the numerical solution of the non-linear thermal conductivity equation. The described models can be applied for the analysis, optimization and practical design of pulsedmode millimetric IMPATT diodes. It can also be used to evaluate the thermal behavior of diodes, to correctly select the shape and amplitude of a supply pulse, and to design various types of high-power pulsed millimeter IMPATT diodes with a complex doping profile with improved characteristics.
\end{abstract}

Key-Words: - Semiconductor microwave devices, modeling and simulation, numerical methods.

Received: January 10, 2021. Revised: June 8, 2021. Accepted: July 4, 2021. Published: July 12, 2021.

\section{Introduction}

IMPATT (IMPact Avalanche ionization and Transit Time) diodes are principal active elements for use in millimetric pulsed-mode generators. Semiconductor structures suitable for fabrication of continuos-mode IMPATT diodes have been well known for a long time [1-2]. They have been utilized successfully in many applications in microwave engineering. The possibilities of using the same structures for pulsed-mode microwave generators are very interesting because the pulsedmode IMPATT-diode generators can successfully operate at high current densities without deterioration of reliability. The cross section of the pulsed-mode IMPATT diode may be larger than that of the continuous-mode diodes. Therefore, the pulsed-mode oscillator can provide a larger power output. Considering, that the increase of the output power of millimetric generators is one of the main problems of microwave electronics; it is important to optimize the diode's active layer to obtain the generator maximum power output.
One of the main singularities in the operation of high-power IMPATT-diode pulsed-mode generator, is the large variation of the diode admittance during the pulse. This variation is significantly during each current pulse due to the temperature changing of the diode's semiconductor structure.

Therefore, diffusion coefficients, ionization rates and charge mobility experience large variations during the pulse. These changes strongly affect the amplitude and phase of the first harmonic of the diode's avalanche current. Therefore, the admittance value also changes. This results in the instability of generator's output power and frequency within each generated microwave pulse.

Pulsed-mode IMPATT diodes that are utilized in microwave electronics are, most frequently, the single-drift and double-drift structures similar to continuous-mode ones [1-5]. The typical diode structure is shown on the Fig. 1 by curve 1, where $N$ is the concentration of donors and acceptors, $l$ is the length of diode active layer. 


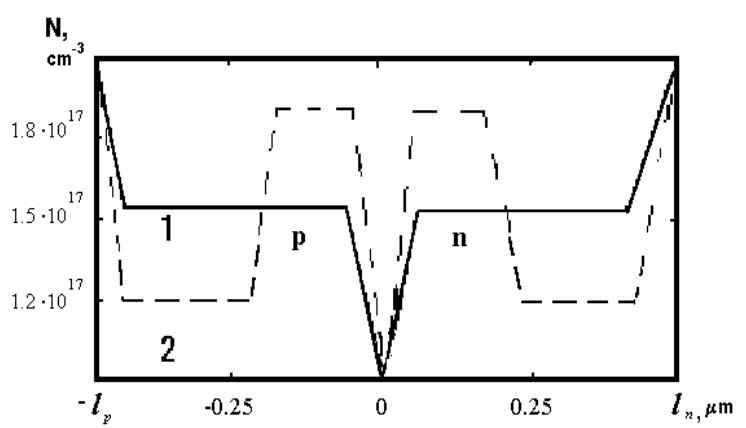

Fig.1. Doping profile for two types of IMPATT diodes: 1 - constant doping profile; 2 - quasi-Readtype doping profile.

In this type of diodes, the electrical field is strongly distorted when the avalanche current density is sufficiently high. This large space charge density is one of the main reasons for the sharp electrical field gradient along the charge drift path. Because of this field gradient, the space charge avalanche ruins itself and consequently the optimum phase relations degrade between microwave potential and current. This factor is especially important when the IMPATT diode is fed at the maximum current density, which is exactly the case at the pulsed-mode operation.

The idea to use a complex doping profile semiconductor structure for microwave diode was originally proposed in the first analysis of IMPATT diode by Read [6]. This proposed ideal structure has never been realized till now. However, a modern semiconductor technology provides new possibilities for the fabrication of sub micron semiconductor structures with complex doping profiles. This stimulates the search for IMPATTdiode special structure's optimization for pulsedmode operation.

The proposed new type of IMPATT diode doping profile is shown on the Fig. 1 by the curve 2 . This type of semiconductor structure can be named as quasi-Read-type structure. This type of doping profile provides a concentration of electrical field within the $p-n$ junction. This measure helps to decrease the destruction of the avalanche space charge and therefore permits to improve the phase stability between the diode current and voltage.

Historically, many analytical and numerical models have been developed for the various operational modes of IMPATT diodes [1, 7-24]. However, they are not adequate for very high current density values and different temperature distributions inside the structure, which is exactly the case for the pulsed-mode IMPATT-diode oscillator. For this reason, we have developed a new complex numerical model of the IMPATT diode that is composed of the advanced thermal model and the modified local-field model. The thermal model provides the exact theoretical temperature distribution along the diode active region. The local-field electrical model calculates the functional dependence of equation coefficients from electric field and temperature, and using all these data finally derives the IMPATT-diode dynamic characteristics.

\section{Numerical Models}

Three numerical models are described in this section. Two different electrical models useful for the precise analysis and internal structure optimization describe all important phenomena into the semiconductor structure. The thermal model describes the temperature distribution by means of the thermal conductivity equation solution.

\subsection{Precise Numerical Model}

The numerical model developed for the analysis of various generator operation modes. This model is based on the system of continuity equations for semiconductor structure:

$$
\begin{aligned}
& \frac{\partial n(x, t)}{\partial t}=\frac{\partial J_{n}(x, t)}{\partial x}+\alpha_{n}\left|J_{n}(x, t)\right|+\alpha_{p}\left|J_{p}(x, t)\right| \\
& \frac{\partial p(x, t)}{\partial t}=-\frac{\partial J_{p}(x, t)}{\partial x}+\alpha_{n}\left|J_{n}(x, t)\right|+\alpha_{p}\left|J_{p}(x, t)\right| \\
& J_{n}(x, t)=n(x, t) V_{n}+D_{n} \frac{\partial n(x, t)}{\partial x} \\
& J_{p}(x, t)=p(x, t) V_{p}-D_{p} \frac{\partial p(x, t)}{\partial x}
\end{aligned}
$$

where $n, p$ are the concentrations of electrons and holes; $J_{n}, J_{p}$ are the current densities; $\alpha_{n}, \alpha_{p}$ are the ionization coefficients; $V_{n}, V_{p}$ are the drift velocities; $D_{n}, D_{p}$ are the diffusion coefficients. Ionization coefficients, drift velocities and diffusion coefficients are functions of two arguments; the spaces coordinate $x$ and the times coordinate $t$.

This model is differs from the previously proposed models because in the present model the ionization coefficients are functions of electric field and temperature in all points of semiconductor structure. The dependence of these coefficients on temperature can be approximated using the approach described in [25]: 


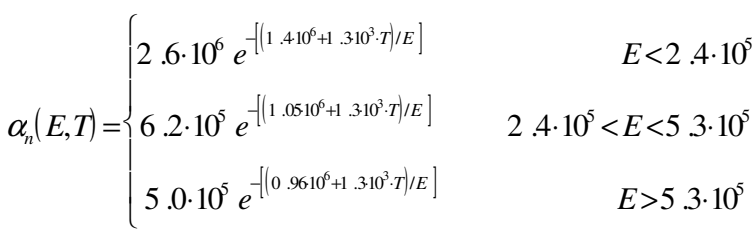

$$
\alpha_{p}(E, T)=\left\{\begin{array}{lr}
\left.2.0 \cdot 10^{6} e^{-\left(1.9510^{6}+1.1 .10^{3} \cdot T / E\right)}\right) & 2.0 \cdot 10^{5}<E<5.3 \cdot 10^{5} \\
\left.5.6 \cdot 10^{5} e^{-\left[\left(1.22610^{6}+1.110^{3} \cdot T / \mid E\right.\right.}\right] & E>5.3 \cdot 10^{5}
\end{array}\right.
$$

The temperature $T$ is expressed in ${ }^{\circ} C$ and electrical field $E$ is expressed in $V / \mathrm{cm}$.

The boundary conditions for the system (1) can be written as follows:

$$
\begin{aligned}
& n(0, t)=N_{D}(0) ; \quad p\left(l_{0}, t\right)=N_{A}\left(l_{0}\right) ; \\
& J_{n}\left(l_{0}, t\right)=J_{n s} ; \quad J_{p}(0, t)=J_{p s} .
\end{aligned}
$$

where $J_{n s}, J_{p s}$ are electron current and hole current for inversely biased $p$ - $n$ junction; $N_{D}(0), N_{A}\left(l_{0}\right)$ are concentrations of donors and acceptors at two space points $\mathrm{x}=0$ and $\mathrm{x}=l_{0}$, where $l_{0}$ is the length of the active layer of semiconductor structure.

Electrical field distribution into semiconductor structure can be obtained from Poisson equation. As electron and hole concentrations are functions of the time, therefore, this equation is time dependent too and time is the equation parameter. Poisson equation for this problem has the following form:

$$
\frac{\partial E(x, t)}{\partial x}=-\frac{\partial^{2} U(x, t)}{\partial x^{2}}=N_{D}(x)-N_{A}(x)+p(x, t)-n(x, t)
$$

where $N_{D}(x), N_{A}(x)$ are the concentrations of the donors and acceptors accordingly, $U(x, t)$ is the potential, $E(x, t)$ is the electric field. The boundary conditions for this equation are follows:

$$
U(0, t)=0 ; U\left(l_{0}, t\right)=U_{0}+\sum_{m=1}^{M} U_{m} \sin \left(\omega m t+\varphi_{m}\right)
$$

where $U_{0}$ is the DC voltage on diode contacts, $U_{\mathrm{m}}$ is the amplitude of the harmonic number $m, \omega$ is the fundamental frequency, $\varphi_{m}$ is the phase of harmonic number $m, M$ is the number of harmonics.

Equations (1)-(4) adequately describe the physical processes in the IMPATT diode in a wide frequency band. However, numerical solution of this system is very difficult because of the sharp dependence of equation coefficients on electric field. The evident numerical schemes have poor stability and require a lot of computing time for the good calculation accuracy obtaining. It is more advantageous to use non-evident numerical scheme, that has a significant property of absolute stability. The computational efficiency and the numerical algorithm accuracy are improved by applying space and time coordinates symmetric approximation.

After the approximation of the functions and its differentials, the system (1) is transformed to the non-evident modified Crank-Nicholson numerical scheme. This modification consists of two numerical systems, each of them having the threediagonal matrix. These systems have the following form:

$$
\begin{gathered}
-\left(a_{n}-b_{n}\right) n_{i-1}^{k+1}+\left(1+2 a_{n}\right) n_{i}^{k+1}-\left(a_{n}+b_{n}\right) n_{i+1}^{k+1}= \\
a_{n} n_{i-1}^{k}+\left(1-2 a_{n}\right) n_{i}^{k}+a_{n} n_{i+1}^{k}+b_{n}\left(n_{i+1}^{k}-n_{i-1}^{k}\right)+ \\
\alpha_{n}\left|\tau \cdot V_{n} \cdot n_{i}^{k}+r \cdot D_{n} \cdot\left(n_{i+1}^{k}-n_{i-1}^{k}\right)\right|+ \\
\alpha_{p}\left|\tau \cdot V_{p} \cdot p_{i}^{k}-r \cdot D_{p} \cdot\left(p_{i+1}^{k}-p_{i-1}^{k}\right)\right| \\
-\left(a_{p}+b_{p}\right) p_{i-1}^{k+1}+\left(1+2 a_{p}\right) p_{i}^{k+1}-\left(a_{p}-b_{p}\right) p_{i+1}^{k+1}= \\
a_{p} p_{i-1}^{k}+\left(1-2 a_{p}\right) p_{i}^{k}+a_{p} p_{i+1}^{k}-b_{p}\left(p_{i+1}^{k}-p_{i-1}^{k}\right)+ \\
\alpha_{p}\left|\tau \cdot V_{p} \cdot p_{i}^{k}-r \cdot D_{p} \cdot\left(p_{i+1}^{k}-p_{i-1}^{k}\right)\right|+ \\
\alpha_{n}\left|\tau \cdot V_{n} \cdot n_{i}^{k}+r \cdot D_{n} \cdot\left(n_{i+1}^{k}-n_{i-1}^{k}\right)\right|
\end{gathered}
$$

$i=1,2, \ldots \quad I_{1}-1 ; \quad k=0,1,2, \ldots \infty$

where $a_{n, p}=\frac{\tau D_{n, p}}{2 h^{2}}, b_{n, p}=\frac{\tau V_{n, p}}{4 h}, r=\frac{\tau}{2 h}, i$ is the space coordinate node number, $k$ is the time coordinate node number, $h$ is the space step, $\tau$ is the time step, $I_{1}$ is the space coordinate node number.

The approximation of the Poisson equation is performed using the ordinary finite difference scheme at every time step $k$ :

$U_{i-1}^{k}-2 U_{i}^{k}+U_{i+1}^{k}=h^{2}\left(N_{D i}-N_{A i}+p_{i}^{k}-n_{i}^{k}\right)$

The numerical algorithm for the IMPATT diode characteristics calculation consists of the following stages: 1) The voltage is calculated at the diode 
contacts for every time step. 2) The initial charge distribution is calculated. 3) The electric potential is calculated at every space point from Poisson equation by the factorization method [26]. The electrical field distribution along the diode active layer is calculated. 4) The ionization coefficients and drift parameters are calculated in numerical net nodes for the current time step. 5) The system (6) is solved by matrix factorization method and electron and hole concentration distribution is calculated for the new time step. After this, the calculation cycle is repeated for all time steps from the beginning to the step 3. This process is continued until the convergence is achieved. The current of the external electronic circuit is determined. Then all harmonics of external current are calculated by the Fourier transformation $\left(J_{0} ; J_{m}=\left|J_{m}\right| \exp \left(j \phi_{m}\right)\right)$; the admittance is calculated for harmonic number $m$ $\left(Y_{m}=J_{m} / U_{m}\right)$ and the power characteristics for all harmonics can be calculated by the following formulas: $\left(P_{m}=-\frac{1}{2} \operatorname{Re}\left(Y_{m}\right)\left|U_{m}\right|^{2} ; \quad \eta_{m}=\frac{P_{m}}{J_{0} U_{0}}\right)$

\subsection{Approximate Numerical Model}

Other numerical model is more suitable for the previous analysis and for the diode internal structure optimization. This model can reduce the total computer time of the structure optimization process.

The numerical method for the solution of the system (1) is based on the classical Fourier series utilization. This approach transforms of the boundary problem for the system of differential partial equations to an ordinary differential equation system. The model describes the physical processes in IMPATT diode by the stationary-operation mode and provides the possibilities to reduce the demands for a computer time that is necessary for the output parameters calculation.

Let us assume that all functions of the system (1) can be presented in a form of Fourier series:

$$
\begin{aligned}
& n(x, t)=\sum_{m=-\infty}^{\infty} n_{m}(x) \cdot \exp (j m \omega t) ; \\
& p(x, t)=\sum_{m=-\infty}^{\infty} p_{m}(x) \cdot \exp (j m \omega t) ; \\
& J_{n}(x, t)=\sum_{m=-\infty}^{\infty}\left(I_{n}\right)_{m}(x) \cdot \exp (j m \omega t) ;
\end{aligned}
$$

$$
\begin{aligned}
& J_{p}(x, t)=\sum_{m=-\infty}^{\infty}\left(I_{p}\right)_{m}(x) \cdot \exp (j m \omega t) ; \\
& \alpha_{n}(x, t)=\sum_{m=-\infty}^{\infty}\left(\alpha_{n}\right)_{m}(x) \cdot \exp (j m \omega t) ; \\
& \alpha_{p}(x, t)=\sum_{m=-\infty}^{\infty}\left(\alpha_{p}\right)_{m}(x) \cdot \exp (j m \omega t) ; \\
& V_{n}(x, t)=\sum_{m=-\infty}^{\infty}\left(v_{n}\right)_{m}(x) \cdot \exp (j m \omega t) ; \\
& V_{p}(x, t)=\sum_{m=-\infty}^{\infty}\left(v_{p}\right)_{m}(x) \cdot \exp (j m \omega t) ; \\
& D_{n}(x, t)=\sum_{m=-\infty}^{\infty}\left(d_{n}\right)_{m}(x) \cdot \exp (j m \omega t) ; \\
& D_{p}(x, t)=\sum_{m=-\infty}^{\infty}\left(d_{p}\right)_{m}(x) \cdot \exp (j m \omega t) .
\end{aligned}
$$

In such a case the principal system (1) can be reduced to a system of the ordinary differential equations for the complex charge density and for the current amplitudes:

$$
\begin{aligned}
& \frac{d n_{m}}{d x}=\sum_{k=-\infty}^{\infty}\left\{-\left(\frac{v_{n}}{d_{n}}\right)_{k} n_{m-k}+\frac{\left(I_{n}\right)_{m-k}}{\left(d_{n}\right)_{k}}\right\} \\
& \frac{d p_{m}}{d x}=\sum_{k=-\infty}^{\infty}\left\{\left(\frac{v_{p}}{d_{p}}\right)_{k} p_{m-k}-\frac{\left(I_{p}\right)_{m-k}}{\left(d_{p}\right)_{k}}\right\} \\
& \frac{d\left(I_{n}\right)_{m}}{d x}=j m \omega n_{m}-\sum_{k=-\infty}^{\infty}\left\{\left(\alpha_{n}\right)_{k}\left(I_{n}\right)_{m-k}+\left(\alpha_{p}\right)_{k}\left(I_{p}\right)_{m-k}\right\}
\end{aligned}
$$

$$
\frac{d\left(I_{p}\right)_{m}}{d x}=-j m \omega p_{m}+\sum_{k=-\infty}^{\infty}\left\{\left(\alpha_{n}\right)_{k}\left(I_{n}\right)_{m-k}+\left(\alpha_{p}\right)_{k}\left(I_{p}\right)_{m-k}\right\}
$$

$m=1,2, \ldots \infty$

where $\left(\alpha_{n}\right)_{m},\left(\alpha_{p}\right)_{m}$ are the electron and hole ionization coefficient amplitudes, $\left(v_{n}\right)_{m},\left(v_{p}\right)_{m}$ are the electron and hole velocity amplitudes, $\left(d_{n}\right)_{m},\left(d_{p}\right)_{m}$ are the electron and hole diffusion coefficient amplitudes, $n_{m}, p_{m}$ are the electron and hole concentration amplitudes, $\left(I_{n}\right)_{m},\left(I_{p}\right)_{m}$ are the electron and hole current amplitudes. 
A number of harmonics $m$ in these series can be reduced down to the number $M$, which defines the accuracy of the solution and necessary computer time. The system (9) can be presented in matrix form as:

$$
Y^{\prime}=A Y
$$

The charge diffusion and sharp dependence of the ionization coefficients on the electrical field determine the great module of eigenvalues of the matrix $A$. For this case, a shooting method, which reduces a boundary problem to Cauchy problem, is not suitable because coordinate basis degenerates in the solution process and therefore is not stable. The boundary problem (10) is solved on the basis of the functional matrix correlation [27] :

$$
B^{t}(x) Y(x)=G(x)
$$

where $B^{t}$ is the factorization matrix; $G$ is the boundary condition vector. The unknown matrixes of equation (11) are satisfied in the following differential equation system:

$$
\begin{aligned}
& B^{\prime}+A^{t} B=0 \\
& G^{\prime}=0
\end{aligned}
$$

The fundamental matrix $F$ is used to obtain the process stability of the integration of equations (10). This matrix is determined as $F(x)=\exp \left\{A^{t}\left(x_{k}\right) h_{k}\right\}$, where $h_{k}$ is the space step. Transition to the next coordinate node is made using the term $B\left(x_{k}+h_{k}\right)=F\left(x_{k}\right) B\left(x_{k}\right)$. The degradation of coordinate basis $B$ can be overcome using the Gram-Schmidt ortogonalization procedure for equation (9) on each integration step.

The algorithm for the analysis of IMPATT diode includes the following steps: 1) the initial charge distribution in the diode is calculated;2) the electric field harmonics are determined from the Poisson equation; 3) ionization and drift parameters are determined from the Fourier analysis, and the matrix of the system of equations on the coordinate net is formed; 4) the boundary problem is solved for the system of continuity equations. Charge and current amplitudes are determined. The harmonics of the external circuit current are calculated. After this, the calculation cycle is repeated from the beginning to point 2) until the external current is determined with sufficient convergence. Then all output parameters of the IMPATT diode are determined.
The main advantage of this harmonic method is the reducing the total computer time for the calculation of stationary mode of the IMPATT diode. In Fig. 2 are shown computer time $T c$ in relative units and relative error $\mathrm{Er}$ as the functions of the harmonic number $M$.

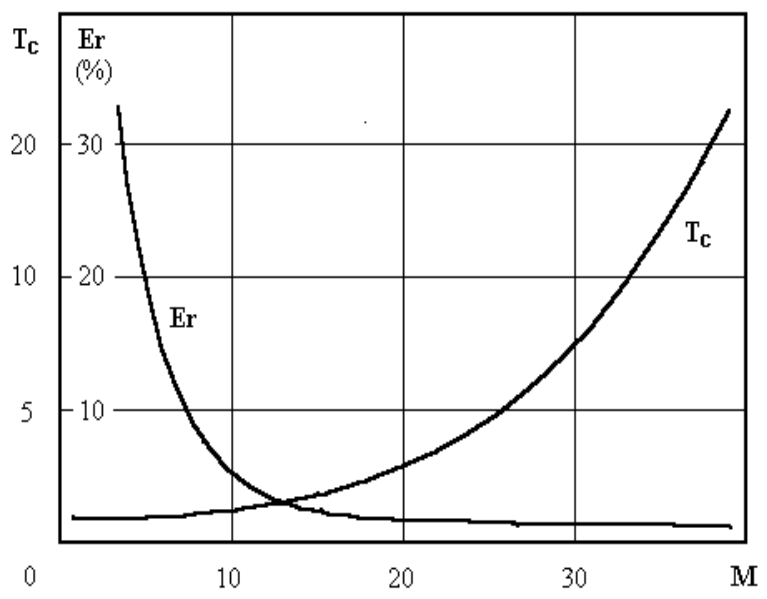

Fig. 2. Computer time $T_{c}$ in relative units and relative error $\mathrm{Er}$ as the functions of the harmonic number $\mathrm{M}$.

These data are corresponded to the nonlinear modes with average level of the non-linearity. For this case we determine error as the relative difference of the diode admittance value that we obtain by this harmonic method and by more precise numerical method of the section 2.1. It is clear that the harmonic number $M$ more than $12-15$ is sufficient to obtain a good accuracy of the diode parameters. At the same time we have a significantly reducing of the total computer time. Computer time for one probe of diode analysis is the principal characteristic of the optimization procedure. That is the main reason why this approximate model is elaborated. For example the total computer time for the diode analysis by precise numerical model is corresponded to the number of harmonic $M=40$.

\subsection{Thermal Model}

The data of the temperature distribution that is necessary for the calculation of the local-field electrical model may be obtained from IMPATT diode thermal model. This model determines the temperature distribution in all points of the diodes active layer, for any given moment in time.

The IMPATT diode thermal model is based on the numerical solution of the non-linear thermal conductivity equation for silicon crystal, contact planes and heat sinks. It determines the instantaneous semiconductor structure temperature 
at any point within the device for any "long" time moment $t^{\prime}$. The thermal equation is solved in the region that is shown on the Fig.3.

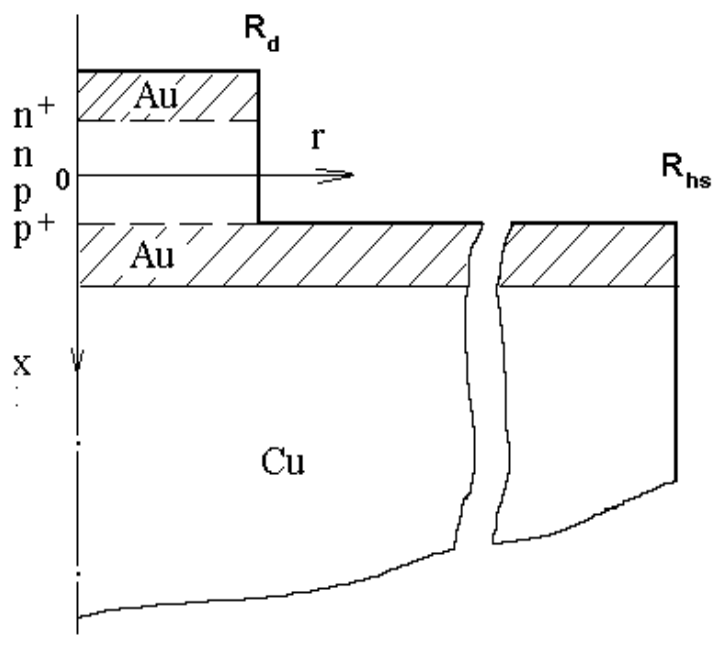

Fig. 3. The schematic diode construction with heat sink. $R_{d}$ - the diode radius, $R_{h s}$ - the heat sink radius.

The thermal equation has the following form:

$$
\frac{\partial T}{\partial t^{\prime}}=\frac{k}{\rho C} \Delta T+\frac{1}{\rho C} Q\left(x, t^{\prime}, T\right)
$$

where $\left(f^{\prime} 1\right)$ is the time coonginate (this time scale differs from the scale in the system (1) ), $r$ is the radial coordinate, $x$ is the longitudinal coordinate, $T$ is the Kelvin temperature; $\rho$ is the material density, $C$ is the specific thermocapacity, $k$ is the thermoconductivity coefficient, and $Q\left(x, t^{\prime}, T\right)$ is the internal heat source that, in the general case, has a dependency on the electrical field, current density, and temperature, $\Delta$ is the two-dimensional Laplace operator and for the cylindrical coordinate system has a form: $\Delta T=\frac{\partial^{2} T}{\partial x^{2}}+\frac{1}{r} \cdot \frac{\partial}{\partial r}\left(r \frac{\partial T}{\partial r}\right)$. The equation (13) is solved within a volume that includes the silicon crystal; the gold contact plane deposited on the crystal; an integrated thermal contact and the semi-infinite copper heat-sink. The boundary conditions for the equation (13) are follows: $\frac{\partial T}{\partial r}=0$ on the vertical axis of symmetry, $\frac{\partial T}{\partial r}=-\lambda(T-\theta)$ on all vertical boundaries facing the air, $\frac{\partial T}{\partial x}=-\lambda(T-\theta)$ on all horizontal boundaries facing the air, $\frac{\partial T}{\partial x}=-\frac{q\left(t^{\prime}\right)}{k} \quad$ on the internal boundary with a semi-infinite copper heat sink. The variable $\lambda$ is the heat transmission coefficient on the metal-air boundary, $\theta$ is the air temperature, $q\left(t^{\prime}\right)$ is the thermal flux entering semi-infinite copper heat sink.

The principal difference between the equation (13) and the system (1) is that: the function $T$ in (13) depends on two spaces coordinates $x$ and $r$. On contrary, all functions of the system (1) depend only on one space coordinate $x$. The dependence of all functions of system (1) on $r$ can be neglected, because of approximations which result in negligible error. However, the same dependence can not neglect for equation (13), because it corresponds really to the two-dimensional case (Fig. 3). We need to determine the functional dependency of the internal heat source $Q\left(x, t^{\prime}, T\right)$ on the temperature to elaborate the IMPATT-diode thermal model. This model may be simplified significantly by the following important approximations: the role of some metal layers (e.g., chromium, gold, palladium) in the diode thermal balance and the influence of gold contact wire and of ceramic housing of IMPATT-diode crystal may be neglected. Also, the heat exchange between diode elements and the atmosphere may be neglected. These simplifications do not seriously affect the accuracy of the model. The internal heat source is defined for all points within the model volume as follows:

$$
Q\left(x, t^{\prime}, T\right)=\frac{1}{2 \pi} \int_{0}^{2 \pi} J\left(\varphi, t^{\prime}, T\right) E\left(x, \varphi, t^{\prime}, T\right) d \varphi
$$

where $\varphi=\omega t, J\left(\varphi, t^{\prime}, T\right)$ is the instantaneous IMPATT diode structure current density value, $E\left(z, \varphi, t^{\prime}, T\right)$ is the electric field intensity in the point $x$ at the time $t^{\prime}$;

This model is essentially different from the model described in [28] because the heat source is described now as the function of the electric field intensity inside the diode structure (14). This improvement is especially important for increasing the accuracy of the temperature distribution calculation of the active layer of the IMPATT diode.

The numerical solution of equation (13) is performed by the finite difference method. This equation is solved by the alternating direction iteration method for each coordinate direction. The second order of the numerical approximation scheme is used more frequently in this case. The 
alternating direction implicit method can be expressed in compact form as:

$\frac{T_{i j}^{s+\frac{1}{2}}-T_{i j}^{s}}{\tau}=\frac{k}{\rho C}\left(\Lambda_{1} T_{i j}^{s+\frac{1}{2}}+\Lambda_{2} T_{i j}^{s}\right)+\frac{1}{\rho C} Q_{j}^{s}$

$\frac{T_{i j}^{s+1}-T_{i j}^{s+\frac{1}{2}}}{\tau}=\frac{k}{\rho C}\left(\Lambda_{1} T_{i j}^{s+\frac{1}{2}}+\Lambda_{2} T_{i j}^{s+1}\right)+\frac{1}{\rho C} Q_{j}^{s+\frac{1}{2}}$

$i=1,2, \ldots I_{2}-1 ; j=1,2, \ldots J-1 ; \quad s=0,1,2, \ldots \infty ;$

where $i, j$ are the space coordinate numbers, $s$ is the time coordinate number, $\Lambda_{1}$ is the partial numerical Laplace operator on the direction $r, \Lambda_{2}$ is the partial numerical Laplace operator on the direction $x$. Two of these operators are defined in the standard five-points numerical pattern:

$\Lambda_{1} T_{i j}=\frac{1}{h_{1} i} \frac{T_{i+1, j}-T_{i-1, j}}{2 h_{1}}+\frac{T_{i+1, j}-2 T_{i, j}+T_{i-1, j}}{h_{1}^{2}}$, $\Lambda_{2} T_{i j}=\frac{T_{i, j+1}-2 T_{i, j}+T_{i, j-1}}{h_{2}^{2}}$. The numerical scheme (15) has the second approximation order only. In this case, it is necessary to develop the numerical net with a large number of cells to obtain sufficient accuracy. That is the reason why the total computer time that is necessary for the solution of the optimization problem is too great. In this work, we propose the other type of thermal equation numerical approximation scheme for the acceleration of the thermal equation solution and for the reduction of the computer analysis time. The total analytic Laplace operator $\Delta T$ can be approximated with the numerical Laplace operator $\Lambda T_{i j}$ as:

$$
\Lambda T_{i j}=\left(\Lambda_{1}+\Lambda_{2}+\frac{h_{1}^{2}+h_{2}^{2}}{12} \Lambda_{1} \Lambda_{2}\right) T_{i j}
$$

In that case, we can approximate the right part of equation (13) by the following numerical formula:

$$
\frac{k}{\rho C} \Lambda T_{i j}+\frac{1}{\rho C}\left(E+\frac{h_{2}^{2}}{12} \Lambda_{2}\right) Q_{j}
$$

where $E$ is the identity operator. The operator $\Lambda$ is defined in the nine-point numerical pattern. The approximation (17) is more complicated, but it has the fourth approximation order. In such a case, we can use the numerical net that is significantly more thin to obtain accuracy that is equal to the scheme (15) described above. For the solution of the principal equation (15) by approximations (16)(17), we used one modification of the PeacemanRachford numerical scheme that had been developed by [29]:

$$
\begin{aligned}
(E- & \left.k \cdot b \cdot\left(\tau-\chi_{1}\right) \Lambda_{1}\right) T_{i j}^{s+\frac{1}{2}} \\
& =\left(E+k \cdot b \cdot\left(\tau+\chi_{2}\right) \Lambda_{2}\right) T_{i j}^{s}+\tau \cdot b \cdot\left(E+\chi_{2} \Lambda_{2}\right) Q_{j}^{s} \\
(E- & \left.k \cdot b \cdot\left(\tau-\chi_{2}\right) \Lambda_{2}\right) T_{i j}^{s+1} \\
& =\left(E+k \cdot b \cdot\left(\tau+\chi_{1}\right) \Lambda_{1}\right) T_{i j}^{s+\frac{1}{2}}+\tau \cdot b \cdot\left(E+\chi_{2} \Lambda_{2}\right) Q_{j}^{s+\frac{1}{2}}
\end{aligned}
$$

where $b=(1 / \rho C), \quad \chi_{1,2}=\left(h_{1,2}^{2} / 12\right)$. We solve the system (16) by the tridiagonal algorithm for radial and longitudinal directions. This numerical scheme provides a significant gain of computer time in comparison with the scheme (15).

The group of the models presented in sections 2.1-2.3 serves as a basis for the precise and complete analysis of the IMPATT diodes with the different doping profiles for the various operation modes.

\section{Numerical Results}

The models described above have been utilized for the investigation of temperature distribution in pulsed mode IMPATT diode having different doping profiles. Also, the diode admittance characteristics have been analyzed. This analysis has been performed for two types of diode structures: for the diode having a traditional constant doping profile, and for the diode having the new special complex doping profile named the quasi-Read type structure. Both of these structures are made of silicon. The first structure has the doping value $N_{0}=1.65 \cdot 10^{17} \mathrm{~cm}^{-3}$ for active layer. The $n$ region length is $0.4 \mu \mathrm{m}$; the $p$ region length is $0.36 \mu \mathrm{m}$. The second structure has two levels of the active layer doping profile: $N_{\text {min }}=1.2 \cdot 10^{7} \mathrm{~cm}^{-3} ; N_{\max }=2.0 \cdot 10^{7} \mathrm{~cm}^{-3}$ and the $n$, $n+, p, p+$ region's lengths are $0.20 \mu m, 0.18 \mu m$, $0.18 \mu \mathrm{m}, \quad 0.16 \mu \mathrm{m}$ respectively. The diode numerical simulation has been performed for the following operational parameters: electrical current pulse has a square form, pulse duration $\tau=100$ nsec, period $\mathrm{T}=10 \mu \mathrm{sec}$. The calculated 
temperature distribution along the diode active layer obtained for pulse current density of 100 $K A / \mathrm{cm}^{2}$ is shown on a Fig. 4 by the solid line for an IMPATT-diode having an even doping profile. The results obtained for the quasi-Read-type structure are also presented in Fig.4 by the dashed line.

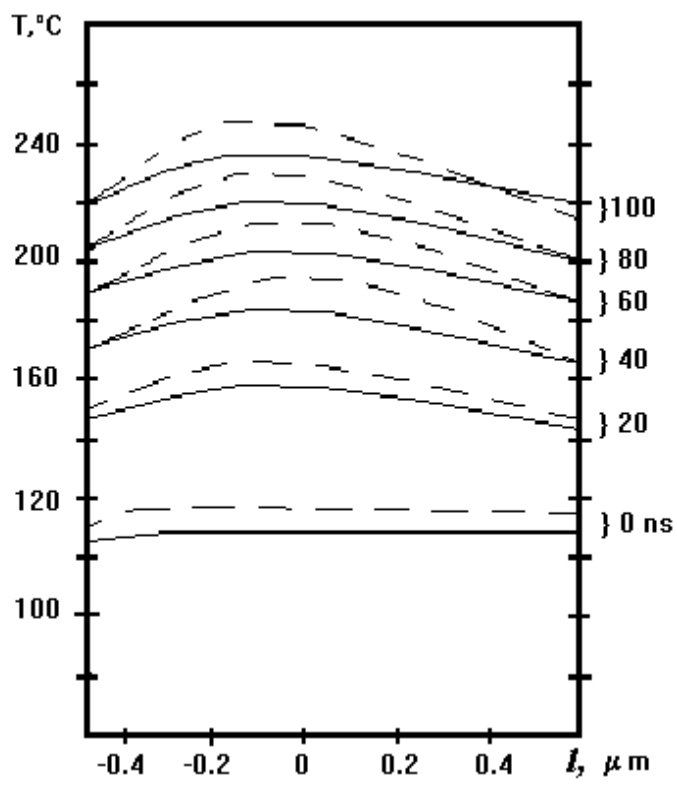

Fig. 4. The temperature distribution.

The data obtained for the diode having complex doping profile demonstrate that the inner part of this diode is hotter than that of the constant doping profile diodes. This occurs because in the complex profile diode electric field intensity maximum is located further from the diode contacts than in the constant doping profile diode. It means that the heat source is located further from the contact regions too and for a such case the complexdoping-profile structure thermal flow dissipates slower than in the first structure. This also explains the existence of a larger temperature gradient along the active layer in the complex doping profile diode. For the structures studied, the temperature difference between the $p-n$ junction and the contacts has been $18,8^{\circ} \mathrm{C}$ for the complex profile diode and $14,4^{\circ} \mathrm{C}$ for even doping profile one.

Data on the diode active layer temperature, obtained for all time step from 0 to $100 \mathrm{nsec}$ has been used for the calculation of the diode dynamic admittance characteristics employing the nonlinear electrical model. Several examples of the calculated admittance characteristics are shown in Fig. 5.

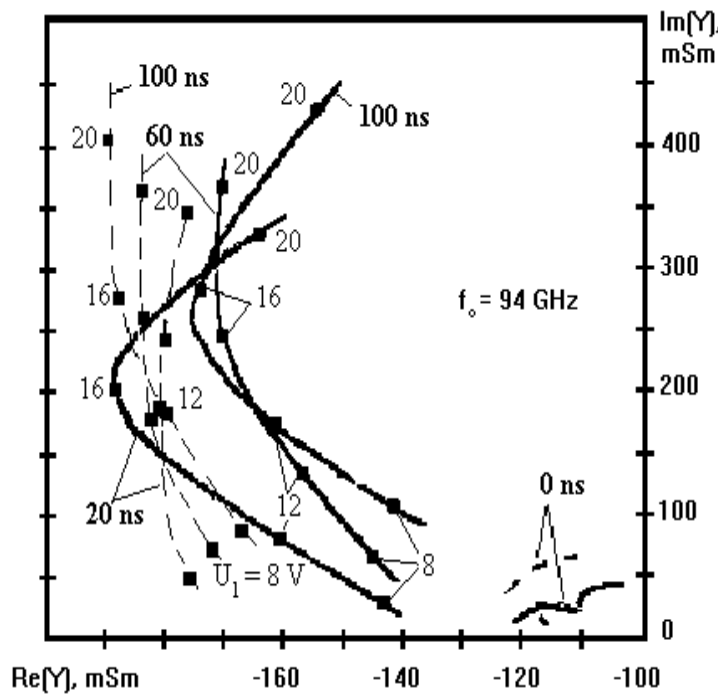

Fig. 5. Admittance frequency characteristics.

These characteristics obtained for the same two types of IMPATT diode doping profiles (solid lines for the even doping profile structure and dashed lines for the quasi-Read structure), and for the same operational modes as described previously.

These diagrams provide complete information on diode admittance variation with time, during the feed pulse. During the first $15 \mathrm{nsec}$ the diode admittance varies very significantly. This variation is due to the strong temperature dependence of the physical parameters of silicon, in the temperature range of $100-170{ }^{\circ} \mathrm{C}$. During the initial part of the feed pulse, the instantaneous active layer temperature falls exactly within this temperature range. During the next $85 \mathrm{nsec}$, the diode admittance has a stable value. It is obvious, that it is impossible to obtain adequate conditions for a stable microwave frequency during the initial part of the current feed pulse, if only special frequency stabilization methods are not utilized. During this initial period of the feed pulse, it is possible to obtain some acceptable frequency stability only by utilizing a special external passive circuit, or by the synchronization of the signal. During the period from 20 to $100 \mathrm{nsec}$, the imaginary part of diode admittance $\operatorname{Im}(Y)$ is changing more rapidly than the real part $\operatorname{Re}(\mathrm{Y})$. Relative variations of $\operatorname{Im}(\mathrm{Y})$ are around $50 \%$ of its average value. However, variations of $\operatorname{Re}(\mathrm{Y})$ are only within $5 \%$ of the average value. This demonstrates that the main cause of the generator's signal instability is the violation of reactive energy balance during the feed pulse. Concurrently, the active energy balance is conserved to a large degree of accuracy. 
In order to provide some means for improving the frequency and amplitude stability of the pulsed IMPATT diode generator, it may be of interest to investigate the influence of the feed current pulse shape on phase relationship between diode current and voltage. Using the present model, the optimum shape of the feed pulse, and the initial and the final feed current density values can be determined for the particular case as functions of the diode material, doping profile, diode structure diameter, etc. However, the comparison of the admittance characteristics for the two types of IMPATT diodes leads to some important conclusions. The data on the diode admittance characteristics presented in the Fig. 5 show that the variations of admittance value during the pulse are much less for the second structure, (dash lines) than for the first (solid lines). This can be observed in Fig. 5, where the admittance curves for the quasi-Read structure lie closer to each other, than the curves displaying the traditional even-profile diode. This can be explained by the larger electric field intensity in the central part of active layer. The larger field intensity results in more compact and dense $n$ and $p$ avalanches propagating in the semiconductor structure and, therefore, in better phase stability between current and voltage. Therefore, the use of the quasi-Read structure, secures better frequency and amplitude stability of the generated electromagnetic oscillations. This is the principal and very important advantage of the new quasiRead structure in comparison to the even doping profile structure.

\section{Conclusion}

The numerical models of the IMPATT diode presented in this paper have important advantages over other models. Electric models together with the thermal model take into account the temperature distribution in the semiconductor structure and the dependence of all basic physical parameters of the semiconductor structure on temperature and electric field. Another important advantage is the high stability of the computational process due to the implicit difference scheme, which is used to solve the basic system of equations.

An approximate nonlinear model of an IMPATT diode can be successfully used to optimize the internal structure. In this case, you can get a great acceleration of the optimization process.

The proposed thermal and electrodynamic models of the local field for the analysis of IMPATT diodes in a pulsed mode make it possible to increase the accuracy of calculating the internal and external characteristics of diodes. The method presented here can be applied to practical design of pulsed millimeter IMPATT diodes. It can also be utilized for diode thermal regime estimation and for the selection of feed current-pulse shape and amplitude. The method is suitable for the design of IMPATT-diode based oscillators, amplifiers and mixers. The most promising application of the models is the development of the pulsed-mode complex-doping-profile high-power millimetric IMPATT diodes with improved characteristics. Comparative analysis of IMPATT diode thermal and electro-dynamic properties performed for two types of the different doping profiles shows that diodes with complex quasi-Read doping profile have better perspectives for the pulsed feed current modulation mode. This special semiconductor structure has better phase correlation between current and voltage and has the smaller variations in the diode admittance. Therefore the complexdoping-profile diodes have improved frequency stability in pulsed-mode operation compared to the traditional IMPATT diodes having a constant doping profile.

\section{References:}

[1] D.L. Scharfetter, and H.K. Gummel, LargeSignal Analysis of a Silicon Read Diode Oscillator, IEEE Trans., Vol.ED-16, No.1, 1969, pp. 64-77.

[2] Edited by M.J.Howes, and D.V.Morgan, Microwave Devices. Devices Circuit Interactions. John Wiley \& Sons, 1976.

[3] T.T. Fong, and H.J. Kuno, Millimeter-Wave Pulsed IMPATT Sourse, IEEE Trans., Vol.MTT-27, No.5, 1979, pp. 492-499.

[4] Edited by Kai Chang, Handbook of Microwave and Optical Components, John Wile \& Sons, Vol.1, 1990.

[5] M. Tschernitz, and J. Freyer, $140 \mathrm{GHz}$ GaAs Double-Read IMPATT Diodes, Electron. Letters., Vol.31, No.7, 1995, pp. 582-583.

[6] W.T. Read, A Proposed High-Frequency Negative-Resistance Diode, Bell System Tech. J., Vol. 37, 1958, pp. 401-406.

[7] M.A. El-Gabaly, R.K. Mains, and G.I. Haddad, Effects of Doping Profile on GaAs DoubleDrift IMPATT Diodes at 33 and $44 \mathrm{GHz}$ Using the Energy-Momentum Transport Model, IEEE Trans., Vol.MTT-32, No.10, 1984, pp.13531361. 
[8] A.M. Zemliak, and S.A. Zinchenko, Nonlinear Analysis of IMPATT Diodes, Vestnik K.P.I., Radiotechnika, Vol.26, 1989, pp. 10-14.

[9] C. Dalle, and P.A. Rolland, Drift-Diffusion Versus Energy Model for Millimetric-Wave IMPATT Diodes Modelling, Int. J. Numer. Modelling, Vol.2, 1989, pp. 61-73.

[10] A.M. Zemliak, and A.E. Roman, IMPATT Diode for the Pulsed-Mode, Izvestiya VUZ Radioelectronica, Vol.34, No.10,1991, pp.1823.

[11] V. Stoiljkovic, M.J. Howes, and V. Postoyalko, Nonisothermal Drift-Diffusion Model of Avalanche Diodes, J. Appl. Phys., Vol.72, 1992, pp. 5493-5495.

[12] M. Curow, Proposed GaAs IMPATT Devices Structure for D-band Applications, Electron. Lett., Vol.30, 1994, pp. 1629-1631.

[13] R.P. Joshi, S. Pathak, and J.A. Mcadoo, HotElectron and Thermal Effects on the Dynamic Characteristics of Single-Transit SiC ImpactIonization Avalanche Transit-Time Diodes, $J$. Appl. Phys., Vol.78, 1995, pp. 3492-3497.

[14] O. Tornblad, U. Lindefelt, and B. Breitholtz, Heat Generation in Si Bipolar Power Devices: the Relative Importance of Various Contributions, Solid State Electronics, Vol.39, No.10, 1996, pp. 1463-1471.

[15] A. Zemliak, S. Khotiaintsev, and C. Celaya, Complex Nonlinear Model for the PulsedMode IMPATT Diode, Instrumentation and Development, Vol.3, No.8, 1997, pp. 45-52.

[16] A. Zemliak, and R. De La Cruz Jimenez, An Analysis of the Active Layer Optimization of High Power Pulsed IMPATT Diodes, Computacion y Sistemas, Edicion Especial, Dec. 2002, pp. 99-107.

[17] A. Acharyya, and J.P. Banerjee, Design and Optimization of Pulsed Mode Silicon Based DDR IMPATT Diode Operating at $0.3 \mathrm{THz}$, Int. J. Engineering Science and Technology, Vol.3, No.1, 2011, pp. 332-339.

[18] D. Chakraborty, P. Bhattacharyya, A. Das, M. Garai, and M. Mukherjee, Design Optimization of High-Power Pulsed Mode IMPATT Oscillator: A Generalised Modelling on Sensitivity Analysis, IJCSET, Vol.1, No.5, 2011, pp. 253-258.

[19] A. Das, D. Chakraborty, M. Mukherjee, P. Datta, and U.C. Ray, Chirp Bandwidth Estimation of Pulsed IMPATT Oscillator at 94 GHz Window: A Simplified Approach, IETE J. Research, Vol.58, No.4, 2012, pp. 266-271.

[20] A. Zemliak, F. Reyes, J. Cid, S. Vergara, and E. Machusskiy, Comparative Analysis of DDR and DAR IMPATT Diodes for Wide Frequency Band, WSEAS Trans. Communications, Vol.12, No.6, 2013, pp. 261270.
[21] A. Zemliak, F. Reyes, J. Cid, S. Vergara, and E. Machusskiy, Analysis of DAR IMPATT Diode for Some Frequency Bands, WSEAS Trans. Circuits and Systems, Vol.14, 2015, pp. 128-133.

[22] P. De, Aspects of WBG 4H-Sic Over Si IMPATT Diode at X Band, IOSR J. Applied Physics, Vol.8, No.3, 2016, pp. 23-26.

[23] D.K. Pattanayak, A.K. Dash, and S.P. Khadanga, Impatt Diode-The Future Source For Terahertz Application-A Review, Int. J. Scientific Research \& Development, Vol.4, No.10, 2016, pp. 724-725.

[24] A. Zemliak, F. Reyes, and E. Machusky, Simulation and Optimization of High Power IMPATT Diodes, Int. J. Circuits and Electronics, Vol.5, 2020, pp. 12-18.

[25] W.N. Grant, Electron and Hole Ionization Rates in Epitaxial Silicon at High Electric Fields, Solid-State Electronics, Vol.16, No.10, 1973, pp. 1189-1203.

[26] V.I. Krylov, V.V. Bobkov, and P.I. Monastyrski, Numerical Methods, Nauka, 1977.

[27] N.S. Bakhvalov, N.P. Zhidkov, and G.M. Kobelkov, Numerical Methods, Nauka, 1987.

[28] L.H. Holway, Transient Temperature Behavior in Pulsed Double-Drift IMPATT Diodes, IEEE Trans., Vol.ED-27, No.2, 1980, pp. 433-442.

[29] A.A. Samarsky, About the Choice of Iteration Parameters for Alternating Direction Method for Dirichlet High order Accuracy Differential Problem, Doklady Acad. Nauk USSR, Vol.179, No.3, 1968, pp. 548-554.

Contribution of Individual Authors to the Creation of a Scientific Article (Ghostwriting Policy)

The authors equally contributed in the present research, at all stages from the formulation of the problem to the final findings and solution.

Sources of Funding for Research Presented in a Scientific Article or Scientific Article Itself No funding was received for conducting this study.

\section{Conflict of Interest}

The authors have no conflicts of interest to declare that are relevant to the content of this article.

Creative Commons Attribution License 4.0 (Attribution 4.0 International, CC BY 4.0)

This article is published under the terms of the Creative Commons Attribution License 4.0 https://creativecommons.org/licenses/by/4.0/deed.en 